\title{
Ischemic stroke in a patient with earlier glioma - delayed effect of irradiation?
}

\author{
Anna Kozak-Sykała', Anna Fiedorowicz ${ }^{2}$, Kinga Kozak', Leon Strządała² \\ ${ }^{1}$ Neurology and Stroke Department, Independent Public Healthcare Centre, Jankowski Regional Hospitaln Przeworsk, \\ Poland \\ ${ }^{2}$ Department of Experimental Oncology, Ludwik Hirszfeld Institute of Immunology and Experimental Therapy, Polish \\ Academy of Sciences, Wroclaw, Poland
}

Kozak-Sykała A, Fiedorowicz A, Kozak K, Strządała L. Ischemic stroke in patient with earlier glioma: a delayed effect of irradiation? J Pre-Clin Clin Res. 2019; 13(2): 79-82. doi: 10.26444/jpccr/109733

\begin{abstract}
Early complications associated with glioma therapy are common and may seriously aggravate the patient's condition. On the other hand, little is known about the late effects of glioma treatment. The case is presented of a 58-year-old woman admitted to hospital with suspected brain tumor recurrence. The patient had been diagnosed 3 years earlier with glioblastoma multiforme. On admission, the patient presented dysarthria, central paresis of nerve VII and left limb flaccid paralysis, which led to the suspicion of tumour regrowth. MR brain scans performed 1 hour after symptom onset were inconclusive. However, MR images acquired 72 hours after admission revealed a picture of fresh vascular, ischemic lesion on the right side of the posterior part of the lenticular nucleus. Physicians should be aware that the risk of brain infarct may be increased in long-term survivals with brain tumours.
\end{abstract}

\section{Key words}

irradiation, ischemic stroke, Glioblastoma multiforme, temozolomide, anaplastic astrocytoma

\section{INTRODUCTION}

High-grade gliomas are a group of aggressive tumours, which include anaplastic astrocytomas (grade III) or glioblastomas multiforme (grade IV). The incidence of gliomas accounts for $2-3$ cases per 100,000 of adults [1], this makes them the most frequent brain tumors. The standard treatment of gliomas comprises mass resection, if possible, chemotherapy with temozolomide and radiotherapy [2]. However, this procedure leads only to 14.6 months of median survival [3] and recurrence is very frequent, often in brain regions remote from the primary lesion. Moreover, intracranial cancer treatment can result in additional neurological deficits which considerably affect the quality of the patient's life. Since postoperative complications are well recognized, the delayed effects of glioma therapy are almost unknown. This is largely caused by the low survival rate of patients with this type of tumour. It has been reported that irradiation can produce delayed neurological deficits. One of the potential causes of these deficits is damage of cerebral vasculature. Diaz et Choi. [4] presented the possible sequence of events leading to vasculopathy in which endothelial cells in the microvessels degenerate and proliferate abnormally. The basement membrane becomes thicker and collagen is accumulated in the lumen. This causes a rapid arteriosclerosis which, in turn, may lead to ischemic stroke.

The case is presented of patient with a history of glioma, who was admitted to hospital 3 years after brain tumour diagnosis, with suspicion of cancer recurrence. The initial tests seemed to confirm this diagnosis (although MR scans were rather inconclusive). However, further MRI-based

Address for correspondence: Anna Kozak-Sykała, Neurology and Stroke Department, Independent Public Healthcare Centre, Jankowski Regional Hospital, Przeworsk, Poland

E-mail: aksyka@gmail.com

Received: 24 January 2019; accepted: 30 May 2019; first published: 10 July 2019 examination revealed an ischemic lesion located in the contralateral hemisphere in relation to previously resected mass.

Clinical summary and pathological findings. Written consent was obtained from the patient for publication of this case report and any adequate images. The 58-year-old woman was admitted to the Neurology and Stroke Department due to left haemiplegia and dysarthria. In the medical history of the family, an ischemic stroke was reported in the patient's mother. The in-patient had been diagnosed 3 years earlier with a left temporal lobe tumou. She had a subtotal surgery of the mass in October 2011. Histopathological examination revealed anaplastic astrocytoma possibly transforming into glioblastoma multiforme (Latin: astrocytoma anaplasticum probabiliter in glioblastoma multiforme veretens), grade III/ IV by the WHO. After surgery, the patient presented a speech disorder of mixed aphasia type, predominantly with semantic disturbances. After undergoing a subtotal surgery, the patient received complementary whole brain radiotherapy (60Gy in 30 fractions). She was also treated with temozolomide in a dose of 120 mg during 42 days. Radio- and chemotherapy were carried out from December 2011 - March 2012. After therapy completion, the patient was under permanent oncological, neurological and neurosurgical controls. When admitted in September 2014, the patient presented normal cardiopulmonary functions, was conscious with fully verbal and logical contact, but complained about headache. On medical examination, lack of significant aberrationsit was reported, heart rate 72 beats per minute, blood pressure 140/90 $\mathrm{mm} \mathrm{Hg}$. The results of peripheral blood morphology and biochemical serum analysis (ions, glycaemia, renal and liver functions, markers of inflammation), and TSH test were within proper ranges. In neurological examination, the dysarthria, central paresis of nerve VII and left limb flaccid paralysis were observed (15 points on the NIHSS 
scale). Electrocardiography (ECG) revealed regular sinus rhythm, left axis deviation and flat $\mathrm{T}$ wave in aVL lead. A MRI of the head with a contrast agent was performed as an emergency procedure (approx. 1 hour after neurological symptom onset) (Fig. 1). This showed a fluid signal intensity of a surgical cavity after subtotal resection of the left temporal lobe tumour, which caused a retraction and widening of the occipital and temporal horns of the left lateral ventricle. The region positioned frontally and medially in relation to surgical cavity, localized in the frontal - temporal border, was visualized as a T1 isointense area with T2 increased signal and FLAIR signal undergoing weak, streaky enhancement.

These observations suggested cancer recurrence. The size of this region was approximately $11 \times 13 \mathrm{~mm}$. Slightly above, in the area of frontal horn of the left lateral ventricle, the $11 \times 4 \mathrm{~mm}$-lesion of decreased $\mathrm{T} 1$ and enhanced T2 signals, with nonsignal streak in the central part, undergoing the contrast ring enhancement was registered, which suggested degenerative changes. The condition of the inpatient gradually improved (decrease of left-lateral paresis and speech disorder) at that time. On the basis of clinical symptoms and MR images, the initial diagnosis was a recurrence of cerebral neoplasm.

The patient received anti-oedema treatment (dexamethason, mannitol) and consulted by neurosurgeon. On the third day after admission, the patient's condition aggravated suddenly, presenting impaired consciousness (10 points on the Glasgow scale), speech problems, aphasia (mainly nominative), dysarthria and left-lateral paralysis (20 points in NIHSS stroke scale). The control MR brain scans, performed on the fourth day of hospitalization (Fig. 2), showed a hyperintense area in T2-dependent and FLAIR images, as well as in diffusion weighted MRI, localized in the right side of the posterior part of lenticular nucleus, with the characteristics of a fresh vascular, ischemic lesion.

As a standard procedure, treatment with acetylsalicylic acid in a dose of $75 \mathrm{mg}$ was employed. In the next days, medical examination was extended to Doppler echocardiography of the carotid and vertebral arteries, which did not indicate any haemodynamically significant disturbances of blood flow. Transthoracic echocardiography showed an impaired diastolic function of the left heart ventricle, but no presence of abnormal structures and heart shunts; an angio-CT of the cerebral arteries revealed a lack of vascular-type malformations, as well as features of thrombosis in the system of cranial venae. An additional diagnostic was performed for identification of abnormalities in the coagulation system (INR, APTT, fibrinogen in normal ranges) and lipid profile (increased levels of total and LDL fraction of cholesterol).

Despite the medical treatment, the condition of the patient did not improve. In the following days, she presented considerable psychomotor agitation, reversalthe of the day-night rhythm and delusions (auditory and visual hallucinations). In an electroencephalographical study, high-voltage, irregular electrical activity of slow waves delta and theta, which overlapped in some cases with the highvoltage irregular activity of the sharp waves, was recorded. The patient was then treated with typical and atypical neuroleptics (Haloperidol, Risperidon) and valproic acid, which reduced the symptoms of paranoid syndrome. In reexamination of the brain (3 weeks after stroke incidence), MR scans revealed an ischemic lesion localized in the deep brain structures on the right side of the brain. This image
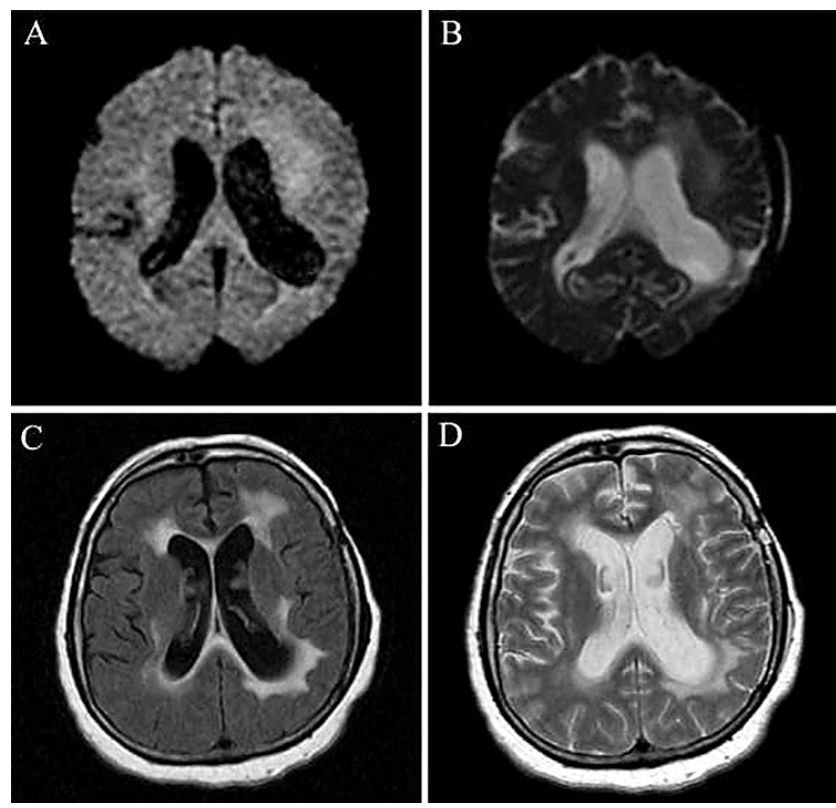

Figure 1. Diffusion-weighted (A, B), fluid-attenuated inversion recovery (FLAIR) (C) and T2-weighted magnetic resonance images (MRI) with gadolinium as contrast agent, recorded 1 hour after symptom onset
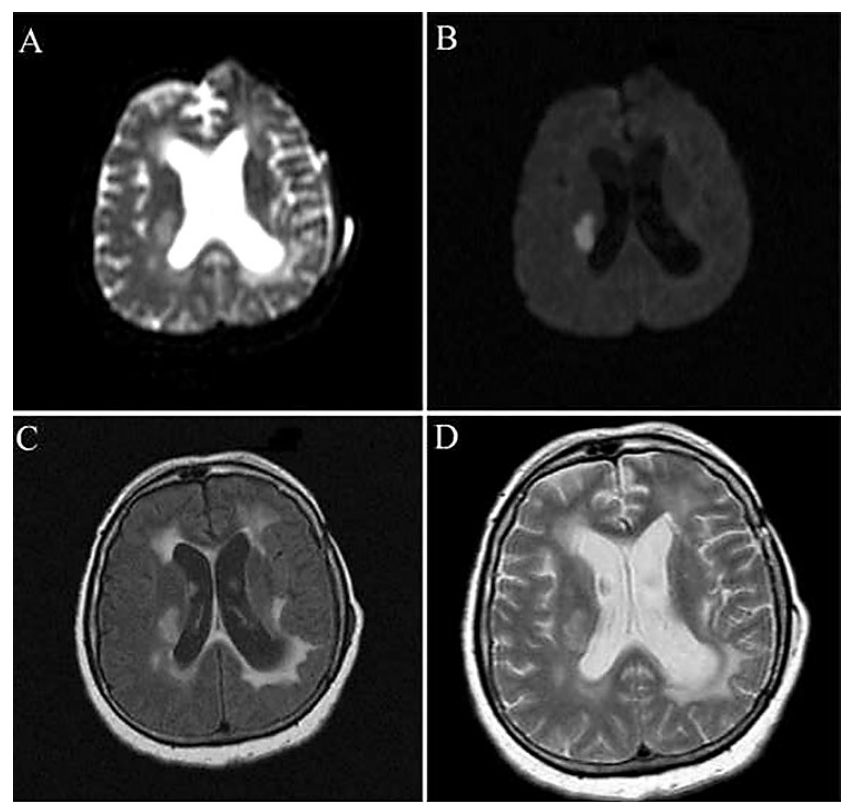

Figure 2. Diffusion-weighted (A, B), fluid-attenuated inversion recovery (FLAIR) (C) and T2-weighted magnetic resonance images (MRI) with gadolinium as contrast agent, recorded 72 hours after symptom onset

did not differ significantly in comparison with that recorded previously. After contrast agent administration, an ischemic area underwent ring enhancement, which reflected reaction on the border of infarcted tissue. Subsequent intensive pharmacotherapy, rehabilitation and speech therapy brought a slight improvement in a clinical condition of the patient.

Four weeks after ischemic stroke, the patient was stable and was moved to the neurological rehabilitation unit. She was fully conscious, but several neurological deficits persisted, such as aphasia, mainly nominative, dysarthria, central paresis of the left facial nerve, left-lateral paralysis (15 points in the NIHSS stroke scale). 


\section{DISCUSSION}

The incidences of ischemic stroke in patients with brain tumours were reported mainly as results of a coexisting cancer or postoperative complications. Kondziolka et al. [5] noted that some brain tumours may manifest as intracranial infarction. This phenomenon is not fully understood and may involve several processes, e.g. neoplasm cell occlusion or tumour-induced blood coagulation changes. Glioblastomas frequently secrete procoagulants, e.g. a tissue factor which may be responsible for thromboembolism observed in such malignancies [6].

In the presented case, the patient experienced an ischemic stroke approximately 3 years after mass removal and radioand chemotherapy. An initial MRI, recorded just after admission, indicated rather cancer recurrence than other causes of neurological symptoms, since early ischemic lesions are barely detectable on MR scans. Similarly, Yaldizli et al. [7] reported the case of patient with ischemic stroke who was diagnosed initially with a brain tumour, based on the neurological symptoms as well as CT scans, which revealed a hyperintense lesion with mass effect and ring-enhancement in the basal ganglia. The suspected tumoral mass turned out to be an ischemic lesion. The cerebral ischemia can be also a serious issue early after brain tumour surgery, but it is mostly unrecognized by the physicians. Wick and Kaufmann [8] state that contrast-enhanced T1-weighted MRI may lead to misdiagnosis of a peri-tumoural infarct when recorded only shortly after mass resection, since a tumour-associated ischemic lesion appears on the MR scan not earlier that 24- $48 \mathrm{~h}$ after the operation, and can be seen during the subsequent several months.

In the patient presented in the current case, the feature of MRI technique was an obstacle in early diagnosis of stroke. In the patient, the brain infarct region was clearly distinguishable on the MR images, 4 days after symptom onset. The glioblastoma multiforme prior to ischemic stroke had already been noted in the literature, both in the case of primary brain tumours and metastases. Two patients who experienced ischemic strokes at the site of glioblastoma development, 3 or 5 months after tumour resections and chemo- and radiotherapy were presented by Rojas-Marcos et al. [9]. Similarly, Obeid et al. [10] noted the case of patient with ischemic stroke secondary to glioblastoma multiforme. As in the previous report, brain infarction occurred only few months after tumour resection, temozolomide treatment and irradiation, again in the region of previous mass presence.

The above-cited examples showed most likely cases of non-radiotherapy-evoked ischemic infarcts, since a too-short delay (a few months) occurred between tumour removal and stroke incidence. In contrast, in the patient presented in the current study, the time interval between cancer operation and stroke was 3 years, thus sufficient to develop therapy-related vasculopathy. The delayed cerebrovascular complications after cranial radiotherapy in adults have already been described [11]. Although data of cohort studies differ in numbers, they are consistent in the general conclusion that cranial radiotherapy increases the risk of stroke, years after treatment. Bitzer and Topka [12] reported that irradiationcaused brain infarcts due to damage to the large vessels appear several years after treatment. Radiotherapy, which is a common procedure in pituitary adenomas, highly increases the risk of cerebrovascular complications, estimated for over
$20 \%$ of all treated patients within a long period of 27 years after radiotherapy [13].

As an illustration of the case report, the example of a woman with breast cancer metastasis to lung and brain can be presented. She was in a complete remission after chemotherapy and whole brain radiotherapy with low doses of irradiation, but 20 years after treatment she experienced a series of ischemic strokes, which were the most probably associated with small vessels pathology [14]. Taking into account the above observations, time of 3 years is sufficient for induction of ischemic stroke by prior radiotherapy in case reported in this manuscript. It was estimated that irradiation administered to the patient was in the $20 \%$ directed to the site of tumour resection and in $80 \%$ to the whole brain. Thus, subsequent ischemic stroke in the brain hemisphere without prior neoplasm, was the most likely caused by the radiationrelated pathological changes in the cerebral vascularization.

It should be mentioned that long-term detrimental effect of temozolomide on the brain vasculature has not been reported in the literature. The possibility exists that ischemic stroke in the currently presented patient was incidental, independent of the preexisting tumour treatment. However, this is unlikely since patient had no considerable risk factors of stroke.

\section{CONCLUSIONS}

In patients with a history of gliomas, the sudden appearance of neurological symptoms usually leads to the suspicion of tumour recurrence. However, earlier therapy, particularly irradiation, may increase the risk of ischemic stroke in longterm survivals due to vasculopathy. Thus, the differential diagnosis with stroke should be performed as soon as possible after patient admission, since the time period from symptom onset to initiation of treatment is crucial in obtaining good outcomes of ischemic stroke therapy.

\section{Acknowledgements}

This study was supported by Grant No. 3/2015 from the State Committee for Scientific Research, Warsaw, Poland.

\section{Conflict of interest}

The authors declare that they have no conflict of interest.

\section{REFERENCES}

1. Verdecchia A, De Angelis G, Capocaccia R. Estimation and projections of cancer prevalence from cancer registry data. Stat Med. 2002; 21: 3511-3526.

2. Urbańska K, Sokołowska J, Szmidt M, Sysa P. Glioblastoma multiforme - an overview. Contemp Oncol (Pozn). 2014; 18: 307-312.

3. Stupp R, Mason WP, van den Bent MJ, Weller M, Fisher B, Taphoorn MJ, et al. Radiotherapy plus concomitant and adjuvant temozolomide for glioblastoma. N Engl J Med. 2005; 352: 987-996.

4. Diaz AZ, Choi M. Radiation-associated toxicities in the treatment of high-grade gliomas. Semin Oncol. 2014; 41: 532-540.

5. Kondziolka D, Bernstein M, Resch L, Tator CH. Brain tumours presenting with tias and strokes. Can Fam Physician. 1988; 34: 283-286.

6. Marras LC, Geerts WH, Perry JR. The risk of venous thromboembolism is increased throughout the course of malignant glioma: an evidencebased review. Cancer 2000; 89: 640-646.

7. Yaldizli O, Kastrup O, Wanke I, Maschke M. Basal ganglia infarction mimicking glioblastoma. Eur J Med Res. 2005; 10: 400-401.

8. Wick W, Kaufmann A. Glioblastoma: what's ischemia got to do with it? Neurology 2006; 67: 1540-1541. 
9. Rojas-Marcos I, Martin-Duverneuil N, Laigle-Donadey F, Taillibert S, Delattre JY. Ischemic stroke in patients with glioblastoma multiforme. J Neurol. 2005; 252: 488-489.

10. Obeid M, Ulane C, Rosenfeld S. Pearls \& Oy-sters: Large vessel ischemic stroke secondary to glioblastoma multiforme. Neurology 2010; 74: e50-51.

11. Dorresteijn LD, Kappelle AC, Boogerd W, Klokman WJ, Balm AJ, Keus $\mathrm{RB}$, van Leeuwen FE, Bartelink H. Increased risk of ischemic stroke after radiotherapy on the neck in patients younger than 60 years. J Clin Oncol. 2001; 20: 282-288.
12. Bitzer M, Topka H. Progressive cerebral occlusive disease after radiation therapy. Stroke 1995; 26: 131-136.

13. Erridge SC, Conkey DS, Stockton D, Strachan MW, Statham PF, Whittle IR, Grant R, Kerr GR, Gregor A. Radiotherapy for pituitary adenomas: long-term efficacy and toxicity. Radiother Oncol. 2009; 93: 597-601.

14. Meijer HJM, Dorresteijn LDA, Schinagl DAX, van Laarhoven HWM. Recurrent stroke after low dose whole brain radiotherapy for brain metastases of breast cancer. IJCRI. 2014; 5: 32-35. 